\title{
Evaluation of the Diabetes Campaign for Palestine Refugees with Diabetes Mellitus Attending UNRWA Health Centers
}

Nada Abu Kishk ${ }^{1 *}$, van den Berg $\mathrm{M}^{2}$, Seita $\mathrm{A}^{3}$, Wafaa Zadian ${ }^{4}$, Turki $\mathrm{Y}^{5}$, Shahin $\mathrm{Y}^{6}$

${ }^{1}$ Health Nutrition Officer- United Nations Relief and Works Agency for Palestine Refugees in the Near East (UNRWA), Jordan.

${ }^{2}$ Research consultant, UNRWA, Jordan.

${ }^{3}$ Director of Health, UNRWA, Jordan.

${ }^{4}$ Health Statistics officer, UNRWA, Jordan.

${ }^{5}$ Health Communication Officer, Health Department UNRWA-Jordan.

${ }^{6}$ Chief Disease Prevention and Control - Health Department UNRWA, Jordan.

\begin{abstract}
Introduction: Among Palestine refugees living in Jordan, Lebanon, Syria, West Bank and Gaza, Diabetes Mellitus Type II (DMII) is a common problem with a prevalence of $11 \%$. A high percent of these patients are obese (64\%) or overweight $(26 \%)$. A DM campaign was conducted to increase awareness on healthy lifestyle and improve health care management of DM among Palestine refugees.

Method: United Nations Relief and Works Agency for Palestine Refugees in the Near East (UNRWA) is the main primary healthcare provider for Palestine refugees. Patients with DMI or DMII for $\geq 1$ year, who were willing to participate, were selected from the 8 largest health centers (HCs) in Jordan, Lebanon, West Bank and Gaza. HCs conducted weekly group sessions for six months, including education, healthy cooking, and physical exercise. Body measurements, 2 hours postprandial glucose tests (2 hr PPGT), blood pressure, cholesterol were collected before and after the campaign.

Result: Of 1,300 included patients, 1,174 (951 females, 223 males) completed the campaign; 36.1\% patients had DMII; $59.3 \%$ DMII and hypertension; and $4.6 \%$ had DMI. After the campaign, $17 \%$ lost $\geq 5 \%$ of their weight, $19 \%$ lost ( $3 \%-5 \%)$, and $29 \%$ lost (1\%-3\%) of their weight. Significant reduction was seen in patients with high-risk waist circumference (WC), ( $\mathrm{p}$-value $<0.001)$. Also, significant improvements were seen in blood sugar and cholesterol.

Conclusion: This campaign focusing on healthy life style awareness by using a variety of group sessions, improved body measurements of Palestine refugees with DM. Such campaigns need to be sustained and expanded to other HCs.
\end{abstract}

Keywords: UNRWA; Palestine Refugees; Diabetes Mellitus; Campaign; Healthy Life Style; Weight Loss; Group Sessions.

\section{*Corresponding Author:}

Nada Mufid Abu Kishk,

Health Nutrition Officer, United Nations Relief and Works Agency for Palestine Refugees in the Near East (UNRWA), Jordan.

Tel: +96265808308

Fax: +96265808319

E-mail: n.abu-kishk@unrwa.org

Received: November 24, 2015

Accepted: December 14, 2015

Published: December 17, 2015

Citation: Nada abu Kishk, et al., (2015) Evaluation of the Diabetes Campaign for Palestine Refugees with Diabetes Mellitus Attending UNRWA Health Centers. Int J Food Sci Nutr Diet. 04(7), 246-252. doi: http:// dx.doi.org/10.19070/2326-3350-1500044

Copyright: Nada abu Kishk ${ }^{\odot} 2015$. This is an open-access article distributed under the terms of the Creative Commons Attribution License, which permits unrestricted use, distribution and reproduction in any medium, provided the original author and source are credited.

\section{Introduction}

The United Nations Relief and Works Agency for Palestine Refugees in the Near East (UNRWA) began operations in 1950. UNRWA has a mandate to provide assistance and protection to a population of around 5 million registered Palestine refugees in Jordan, Lebanon, Syria, West Bank and the Gaza Strip. UNRWA human development and humanitarian services encompass education, primary health care, relief and social services, infrastructure and camp improvement, and emergency response in situations of armed conflict. UNRWA is the main primary healthcare provider for Palestine refugees and it is funded almost entirely by voluntary contributions [1].

In 2013, UNRWA conducted a DM campaign, "Life is Sweeter with Less Sugar" to increase healthy lifestyle awareness and improve health care management of DM among Palestine refugees. Patients with DMI or DMII \& hypertension for $\geq 1$ year, and willing to participate were randomly selected from the 8 largest health centers in the five fields of operations, total 30. Health Centers conducted weekly group educational, healthy cooking, and exercise sessions for six months. Body measurements, blood tests and patients' attendance were collected on monthly basis.

UNRWA has been providing health care for patients with diabetes and hypertension in its health centres since 1992. Diabetes care includes screening of high-risk groups, diagnosis and treatment. Treatment includes promoting a healthy lifestyle and medical assistance such as insulin therapy. A total of 122,400 diabetic patients were registered with UNRWA health centres in 2013 in five 
fields [2]. $17.3 \%$ of total registered patients in UNRWA health centres are diabetic. In 2013, 35\% of UNRWA's total medications budget (USD 22.25 million) was spent on drugs to treat diabetes and high blood pressure [2].

Worldwide, every seven seconds one person dies from diabetes; over 387 million people are currently living with the disease, globally, 612 USD billion is spent on diabetes treatment [3]. As in other regions, one of the leading causes of mortality and morbidity among Palestine refugees are non-communicable diseases (NCD), especially diabetes mellitus Type II (DM II).

Obesity is recognized as an important risk factor for DM II, as it induces insulin resistance and pancreatic beta-cell dysfunction these obesity-related defects tend to progress following weight gain and can eventually lead to worsening hyperglycaemia over time. Evidence from large-scale population studies strongly supports this close association, identifying obesity as one of the most important risk factors for DMII [4]. The Look AHEAD (Action for Health for Diabetes) cohort done by Edward et al. showed that with intensive lifestyle intervention, participants lost significantly more weight than control group participants who only received education [5].

In order to address challenges of diabetes care at UNRWA health centres, the agency had performed a clinical audit with goals to acquire information on current diabetes care in UNRWA health centres [6]. During UNRWA's clinical audit in 2012, it was revealed that over $90 \%$ of DM patients are overweight or obese, with the majority $(64 \%)$ being obese. Based on UNRWA's clinical audit recommendations, many action steps have been agreed, such as: developing a comprehensive package of lifestyle support activities that can be implemented in health centres with virtually no additional cost and UNRWA should change the involvement of nurses and paramedical staff in diabetes care delivery to a more proactive way, in order to improve health promotion, education and adherence [6].

Responding to the above reality UNRWA embarked on a campaign titled "Life is Sweeter with Less Sugar" to assist both patients and staff in changing their attitude and behavior in order to prevent and control DM. The purpose of this campaign was to improving the capacity of health staff for diabetes care management, and to promote awareness about diabetes care among DM patients, through conducting variety of group sessions for 6 months, the group sessions included physical exercise, healthy cooking and education about disease management.

\section{Methodology}

Non-control interventional descriptive study, which we conducted as an awareness campaign, has took place in UNRWA health centers across the four fields (Jordan, the West Bank, Lebanon and Gaza). Three interventional aspects of diabetes care were included: exercise, healthy diet and disease management. The study lasted for 6 months.

\section{Patient enrollment and health centers selected}

For the DM campaign sampling, we followed the same procedure used in the clinical audit [6]. In the clinical audit 50 patients were selected with diabetes from the 8 largest health centers in each of Gaza, Jordan, Lebanon and West Bank. We aimed toenroll 50 patients with diabetes from each health centers in the mentioned four fields. However, because of logistic constraints, we chose 6 health centers instead of eight in Lebanon, and 25 patients instead of 50 patients per health centers in West Bank.

No control group was selected for this study. UNRWA is not an academic institution, and has the mandate to provide health care for its patients. If we have had chosen to include a control group, who would receive standard care only, the possible effect of the diabetes campaign could potential be due to placebo effect. Just by coming to the health centers regularly and more attention for the patient the outcome may improve. We considered it to be unethical to include DM patients in the study, and ask them to come to the clinic regularly for measurements, while no treatment or interventions provided.

Inclusion \& Exclusion Criteria: The following criteria were required for patient enrolment:

- Patients attending the selected health centers with confirmed ${ }^{1}$ Diabetes Mellitus, both DMI (with or without HTN) and DMII (with or without HTN) and had received care at UNRWA NCD clinics for at least one year.

- Patients were willing and committed to participate in all planned activities, and gave a verbal informed consent.

Exclusion criteria: Non-Palestine refugee with DM or Palestine refugees who are not registered in UNRWA health centers.

\section{Sampling}

A total of $1300 \mathrm{DM}$ patients from the 8 largest health centers in Jordan, Lebanon, West Bank and Gaza were randomly ${ }^{2}$ selected to be part of the campaign activities. Before starting the activities, all selected health centers were provided with posters and brochures to promote for the launch of campaign and its activities.

\section{Activities conducted}

Taking into consideration the characteristic of an UNRWA health Centre, such as: long waiting time for patients, short consultation times with doctors, and multiple stops within the health centre. Headquarters health department and all field offices expect for Syria field, have went intensively thru the preparation steps of the campaign, from inception, implementation, to evaluation. Before the campaign started, the following steps were taken, as the campaign took place from January 2013 to January 2014:

1. Pre-planning and preparation in each field by concerned staff with collaboration of UNRWA HQ health department.

2. UNRWA Technical staff at HQ along with diabetes specialties has jointly conducted the training for UNWRA health staff. These training included topics on how to appropriately respond to the needs of patients currently living with diabetes and how to manage their disease.

3. Meetings with the high members of the community in each camp and partnership with local NGOs.

4. Ensuring the availability of funds for each health center.

1. Confirmed in UNRWA HCs by certain criteria (two positive test of $2 \mathrm{hr}$ PPG $\geq 180$ )

2. Randomly selection but not the systematic random method, it was based on the patients welling to participate. Patient attended HC, then was randomly asked if they are willing to participate by medical officer, verbalconsent was given and then were included 
Health centers conducted a variety of group sessions in an as interactive and exciting way as possible. As such, based on the time schedule of every health centers, the following three main activities were conducted on regular basis in every health centers:

1. Education sessions were conducted on average of twice a month. The main purpose was to improve the diabetic patient's knowledge of diabetes management considering: diagnosis, symptoms, risk factors, medication, lifestyle, complication, foot care, late complication, and dental care.

2. Cooking sessions were conducted on monthly basis. Partnership with community kitchens was established to teach and practice healthy ways of cooking of traditional Palestinian recipes. The participant and the medical staff along with the local partners cooked Palestinian recipes in a healthily way, and have tasted the food during the sessions.

3. Exercise sessions existed in the local NGOs, UNRWA schools and health centers on average of twice a month. UNRWA sport teachers and local physical trainers have volunteered to help the medical staff in conducting the sessions.

\section{Data collection}

Demographic information, body measurements and a blood sample were collected at baseline, and on a monthly basis, for up to 6 months. Data was recorded in a standardized Excel sheet with a specific code for each patient.

1. Demographic information: age, gender, DM category (DMI, DMII or DMII \& HTN) and control status.

2. Body measurements: height $(\mathrm{cm})$, weight $(\mathrm{kg})$, and waist circumference $(\mathrm{cm})$ measured from the umbilicus.

3. Blood tests: 2 hours postprandial glucose tests ( $2 \mathrm{hr}$ PPG), total Cholesterol.

4. Blood pressure ( $\mathrm{mmHg}$ ).

5. Attendance for each patient was recorded in every session.

Questionnaires on knowledge and practice of diabetes care were also administrated, before and after the campaign (6 months).

\section{Data Handling and Statistical Analysis}

Data was entered in Excel and thereafter transferred into SPSS version 21.0, which was used for the statistical analysis. Patient demographics, disease pattern and knowledge/behavior before and after the campaign were evaluated descriptively.

The differences between baseline and the end of the campaign in weight, waist circumference, BMI, weight-height ratio, PPGT, Cholesterol and blood pressure were assessed using the paired T-test. This was performed for the complete sample as well as for different patient categories, which were classified according to WHO guidelines for BMI and Waist circumference. Baseline waist circumference for male was: normal $<94 \mathrm{~cm}$, risk $94-102 \mathrm{~cm}$ and high risk $\geq 102 \mathrm{~cm}$ and for female normal $<80 \mathrm{~cm}$, risk 80-88 $\mathrm{cm}$ and high risk $\geq 88 \mathrm{~cm}$. Weight change was categorized as the following classification: weight reduced by: $(\geq 5 \%$, or $3 \%-5 \%$, or $1 \%-3 \%$ ), weight increased by: $(\geq 5 \%$, or $3 \%-5 \%$, or $1 \%-3 \%$ ) or no change by $(+1 \%$ to $-1 \%)$. Baseline BMI was categorized as $<$ 30 (non-obese) or $\geq 30$ (obese). Baseline Weight-to-Height Ratio (WHtR) for males was categorized as $>63$ or no $\leq 63$, WHtR for female as $>58$ or $\leq 58$. Baseline PPGT was $\leq 180 \mathrm{mg} / \mathrm{dl}$ (normal) or $>180 \mathrm{mg} / \mathrm{dl}$ (abnormal). Cholesterol baseline was classified as $<200 \mathrm{mg} / \mathrm{dl}$ (normal) or $\geq 200$ (abnormal). Systolic and diastolic pressure were considered hypertension when $\geq 140$ $\mathrm{mmHg}$ and $\geq 90 \mathrm{mmHg}$, respectively.

\section{Results}

\section{Patient demographics}

The sample size for the DM campaign participants was 1,300 patients. However, 1174 completed the six months of the campaign, of those 1174 that completed the campaign, 964 reported with full data, and 210 had some missing data. Figure 1 describes the number of patients throughout the campaign.

Patient Age and Sex: The average age of patients was $51.9 \pm$ 10.3 years, with $72 \%$ between $40-59$ years of age $(60 \%$ female and $11.8 \%$ male). In all age categories most participants were females $(81.0 \%$; 950 out of 1174$)$.

Figure 1. Number of patients throughout the campaign.

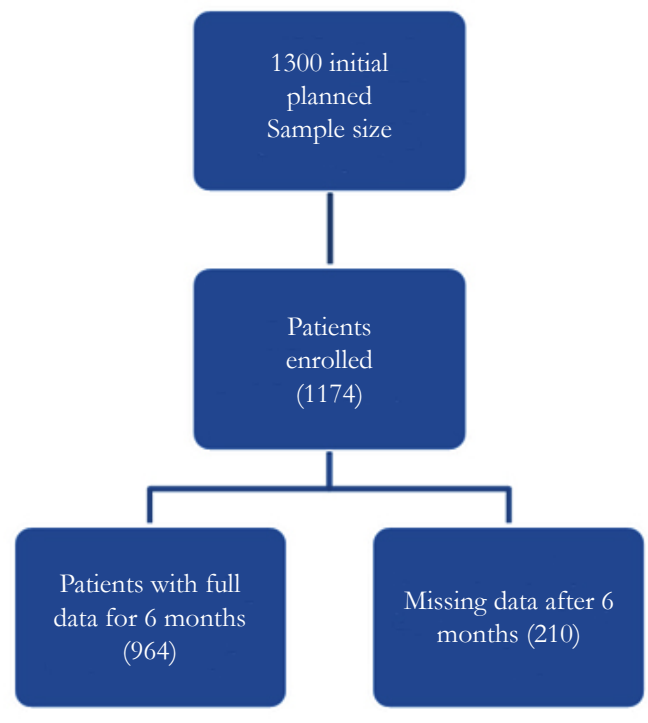


Disease Pattern: Participants were categorized according to their DM/HTN status. A total of $54(4.6 \%)$ of them had DMI, $422(36.1 \%)$ had DMII, and $694(59.3 \%)$ had both conditions of DMII \& HTN. This disease distribution pattern was similar to that found during the UNRWA clinical audit in 2012 [6].

\section{Body measurements}

Changes of outcomes were observed, which resulted from the Analysis of Variance (Paired-Sample T -Test) for all body measurements. Table 1 describes the changes of body measurements from baseline to the end of the campaign.

Body weight: After the 6-month campaign $1.9 \mathrm{~kg}$ was the average weight loss (-2.0 kg for women and $-1.7 \mathrm{~kg}$ for men). While the mean for both genders before and after the campaign was as follow: $(87.3 \pm 17.2$ and $85.5 \pm 16.8)$ respectively, with 1168 of complete weight measurement.

Of all patients who completed the campaign, $17 \%$ lost $\geq 5 \%$ of their weight, $19 \%$ lost $3 \%-5 \%$, and $29 \%$ lost $1 \%-3 \%$ of their weight. However, $20 \%$ had no major change in their weight $(-1 \%$ to $+1 \%$ ), and more than $10 \%$ had an increase in their weight. Figure 2 shows the average weight change in Kgs before and after the campaign for male and female.

Body Mass Index (BMI): On average, BMI showed the reduction of $-0.7 \pm 1.3$ after the 6 -month campaign: it was $-0.8 \pm 1.4$ for female and $-0.6 \pm 1.2$ for male. A significant change was detected in the BMI category of those who are obese $(\mathrm{BMI} \geq 30$ ) (reduced from $36.7 \pm 5.0$ to $35.9 \pm 5.0$ ), and those who are nonobese $(\mathrm{BMI}<30)$, (reduced from $26.8 \pm 2.9$ to $26.6 \pm 2.8$ ). Figure 3 describes the percentage of change in all BMI categories before and after the campaign for both genders.

Waist circumference: From the 1141 with full WC data, it was shown that waist circumference significantly reduced $108.5 \pm 13$ to $106 \pm 13.2$ as described in Table 1. According to World Health Organization (WHO), a waist Circumference (WC) of $\geq 102 \mathrm{~cm}$ in men, or $\geq 88 \mathrm{~cm}$ in women, has a substantially increased risk of health problems such as DMII, heart disease and high blood pressure [7]. Table (2) shows the reduction in WC for the highrisk group, before and after the campaign in both genders.

\section{Biomarkers before and after the campaign}

Table (3) shows the PPGT results of 1139, the Cholesterol results of 1122, and blood pressure readings of 1146 participants, before and after the campaign. A significant reduction was detected for those who were above the normal cutoff of point PPGT $>180$ before $253.7 \pm 64.5$ after $196.3 \pm 72.8$, cholesterol $>200$ before $235.4 \pm 32.7$ after $209.6 \pm 38.7$ and BP (systolic/diastolic) $\geq$ $140 / 90)$ before $149.4 \pm 11.3 / 93.3 \pm 5.6$ after $130.4 \pm 14.4 / 81.4$ $\pm 9.6$

However, an increase in the biomarkers average was detected for those who are within or below the normal cutoff points, which are 2 hr PPGT $\leq 180$, cholesterol $\leq 200$, BP: $<140 /<90$, that might be for several reason including day-to-day variation, measurements error, etc.

\section{Patients' behavior and knowledge before and after the cam- paign}

Based on the pre-post questionnaires, improvements were observed in the knowledge and lifestyle practices among the 1174 patients. For example, an improvement was seen in the cooking practices, specifically when the patients were asked about the way he/she adds oil during cooking? There was a significant shift from estimating the amount of oil added during cooking, to using a measurement tool in order to add oil during cooking.

Figure 2. The Average weight change before and after the campaign for male and female.

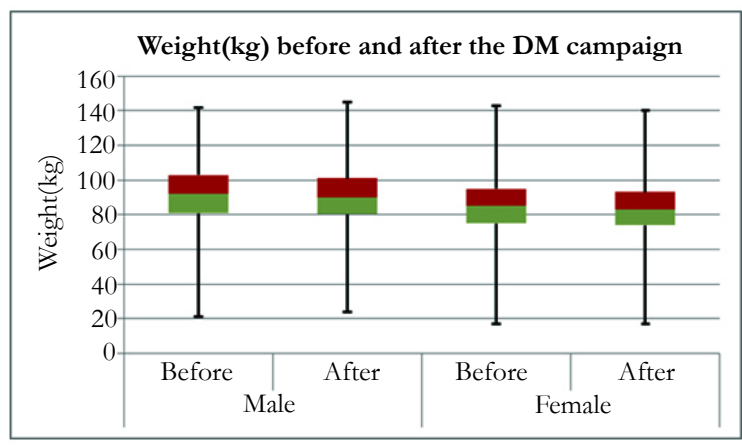

Figure 3. BMI categories before and after the campaign for both genders.

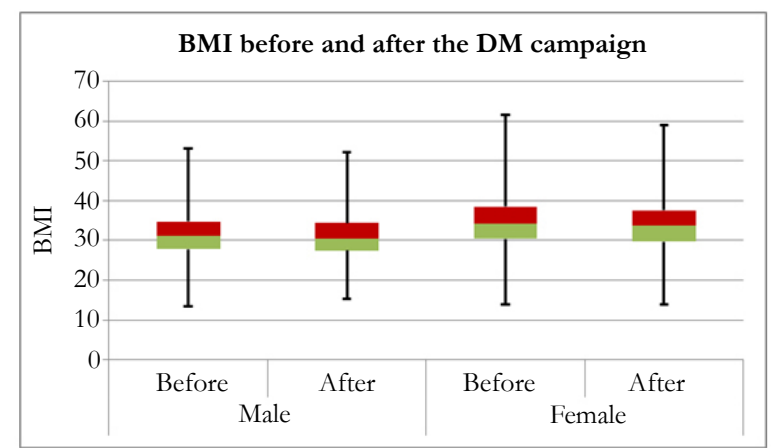


Table 1. Change of body measurements from before and after the campaign.

\begin{tabular}{|c|c|c|c|c|}
\hline \multirow{2}{*}{ Parameters } & Before campaign & After campaign & \multicolumn{2}{|c|}{ Changes } \\
\cline { 2 - 5 } & Mean \pm SD & Mean \pm SD & Mean \pm SD & p - value \\
\hline Weight $(\mathrm{Kg})$ & $87.3 \pm 17.2$ & $85.5 \pm 16.8$ & $-1.9 \pm 3.4$ & $<0.001$ \\
\hline Waist circumference $(\mathrm{cm})$ & $108.5 \pm 13.1$ & $106 \pm 13.2$ & $-2.6 \pm 4.9$ & $<0.001$ \\
\hline Body mass index $\left(\mathrm{Kg} / \mathrm{m}^{2}\right)$ & $34.1 \pm 6.3$ & $33.4 \pm 6.2$ & $-0.7 \pm 1.3$ & $<0.001$ \\
\hline Body mass index - male & $31.6 \pm 5.9$ & $31.0 \pm 5.8$ & $-.6 \pm 1.2$ & $<0.001$ \\
\hline Body mass index - female & $34.6 \pm 6.3$ & $33.9 \pm 6.1$ & $-.8 \pm 1.4$ & $<0.001$ \\
\hline
\end{tabular}

Table 2. High risk waist circumference before and after the campaign in both genders.

\begin{tabular}{|c|c|c|c|c|}
\hline Waist circumference (WC) & Categories & WC mean before & WC mean after & P value \\
\hline WC - Male & $\geq 102$ & $114.3 \pm 8.6$ & $111.9 \pm 8.7$ & 0 \\
\hline WC - Female & $\geq 88$ & $110.2 \pm 11.2$ & $107.4 \pm 11.7$ & 0 \\
\hline
\end{tabular}

Table 3. Biomarkers before and after the campaign.

\begin{tabular}{|c|c|c|c|c|}
\hline \multirow{2}{*}{ Parameters } & Before campaign & After campaign & \multicolumn{2}{c|}{ Changes } \\
\cline { 2 - 5 } & Mean \pm SD & Mean \pm SD & Mean \pm SD & p - value \\
\hline Systolic blood pressure $(\mathrm{mmHg})$ & $128.1 \pm 16.5$ & $124 \pm 3.8$ & $-4.1 \pm 17$ & $<0.001$ \\
\hline Diastolic blood pressure $(\mathrm{mmHg})$ & $78.6 \pm 9.3$ & $77.5 \pm 8.1$ & $-1.1 \pm 10.1$ & $<0.001$ \\
\hline Cholesterol $(\mathrm{mg} / \mathrm{dl})$ & $195.9 \pm 44.3$ & $188.4 \pm 40.7$ & $-7.2 \pm 40.3$ & $<0.001$ \\
\hline 2-hr PPG $(\mathrm{mg} / \mathrm{dl})$ & $213.1 \pm 79.1$ & $181 \pm 70.4$ & $-32.1 \pm 83$ & $<0.001$ \\
\hline
\end{tabular}

Also, a significant increase in the number of meals consumed thru the day were observed, the percentage of having 5-6 meals per day was significantly increased from $4.6 \%$ (54 patients) to $16.1 \%$ (181 patients), which we think it might be a result from healthy messages given from the staff in the educational and cooking sessions on how importance the small and frequent meals with low fat in managing the blood glucose and losing weight.

\section{Patients' attendance during the campaign}

The percentage of attendance was quite similar in the sessions conducted during the campaign: $83.3 \%$ (1011) for education, $84.8 \%$ (1010) for cooking, and 83.2\% (1014) for exercise sessions. These figures are considered reasonably high, especially given the poor camp settings, infrastructure and facilities provided for the campaign activities.

\section{Discussion}

Globally, many efforts towards healthy lifestyle changes for patients with diabetes have been made. Many studies have shown that patients with diabetes do not adequately manage their disease, even after receiving education about diabetes care [8-10].

Deakin et al, have shown that Group-based training for selfmanagement strategies in people with DMII is effective by improving fasting blood glucose levels, glycated haemoglobin and diabetes knowledge and reducing systolic blood pressure levels, body weight and the requirement for diabetes medication [11].

The diabetes campaign "life is sweeter with less sugar" which UNRWA conducted by using a variety of interventions, was found to be a relevant awareness tool. In our experience, one of the main reasons why the campaign appeared to have a positive effect on patient outcomes and was welcomed by the local community is that staff took ownership of the campaign. Almost every staff member in the health centre participated in conducting campaign activities to some extent; this collaboration and teamwork led to improved cooperation within the health center, and may have influenced the improved outcomes seen by the patients.

Patient's attendance level was considered reasonably high, especially given the poor camp settings; infrastructure and facilities provided for the campaign activities, but it might be from the social bonding that happened during the sessions, as the activities were interesting and attractive for the participants. We experienced that many positive results could be due to social gathering during the education, exercise and cooking sessions. Also with the variety of sessions and activities in delivering the messages, more adherences were seen because patients experience that they were not alone with their disease and problems and they are not depressed. However, the new struggle in light of these achievements is how to sustain the positive outcomes of such interventions.

Weight loss with diet and exercise regimens has been shown to effectively improve glycemic control. Importantly, it has been shown that in patients with DMII even a modest sustained reduction of the initial body weight (5-10\%) can significantly mitigate diabetes-related complications by improving glycemic control, lipid profiles and blood pressure. In more recent meta-analysis of randomized controlled trials, it was demonstrated that lifestyle interventions can result in a modest pooled weight loss of 1.7 $\mathrm{kg}(3.1 \%$ of body weight $)$ in DMII adults, with improvements in HbA1C 2.6\% [12]. Comparing these results with UNRWA DM campaign, it was found that $17 \%$ of patient have reduced $\geq 5 \%$ of their weight, $19 \%$ have lost $3-5 \%$, and $29 \%$ have reduced $1-3 \%$ of their weight, with an average of $1.9 \mathrm{~kg}$ decrease in weight. However, maintaining the reduction of weight can be difficult, especially after the campaign activities are finished. To prevent weight regain after the campaign, a sustainable follow-up of the patient is being conducted.

Waist circumference has been recognized as an independent risk 
factor for DMII, it was found to be the best anthropometric predictor of visceral fat and abdominal fat. WC of $\geq 102 \mathrm{~cm}$ for male and $\geq 88 \mathrm{~cm}$ for female, are considered to indicate high risk of obesity-related health problems, DM, cardiovascular diseases and other chronic diseases $[13,14]$. Therefore, it was important to measure the changes in WC before and after the campaign, which was significantly decreased $(2.4 \mathrm{~cm} \pm 4.9)$. Another studies (Deakin, et al., [11]) conducted group based education sessions, resulting in no significant difference between the two intervention groups at four months (difference $1.3 \mathrm{~cm}$ ) but there was a trend in favor of the group education program at 14 months (difference $2.8 \mathrm{~cm}$ ).

2 hrs PPG shows how tolerant the body is to glucose; therefore it is a one-time level of blood glucose only. UNRWA uses $2 \mathrm{hr}$ PPG results for diabetes monitoring and control. As per UNRWA Technical Instructions [3], diabetes control is achieved when 2 $\mathrm{hr}$ PPG becomes equal or less than $180 \mathrm{mg} / \mathrm{dl}$ ( $\leq 10.0 \mathrm{mmol} / \mathrm{L})$. Based on that, 2 hrs PPG was used as monitoring tool during the campaign, which resulted in a significant reduction before and after the campaign. However, patients can impact results by not eating 2 hours before the test, heavy exercise, or having a meal or a snack right before the test [15]. During UNRWA's clinical audit [7], it was shown that the diabetes control rate among UNRWA patients as a result of $\mathrm{HbA1c}$ tests is lower than that measured using 2-hr PPG (28\% vs. 45\%). Thus, estimating diabetes control through 2-hr PPG may be misleading as it over-estimates control rates. In light of the financial constrains that UNRWA is facing, UNRWA will develop a stepwise implementation plan for the introduction of modern technologies (HbA1c) for patients, who need and would benefit from it the most.

The results of such a campaign indicate that using an existing NCD care program embedded in UNRWA primary health centers is a feasible way to implement combined diabetes care management and weight loss. The adaptation of variety of focus group sessions, such as exercise, cooking and education sessions, with local NGOs and community partnership, have shown to a positive result in reducing weight, WC, 2 hr PPGT, Cholesterol and BP.

\section{Limitations}

Being a non-control descriptive study with purpose of increasing awareness and not for academic purposes, it has resulted in data discrepancies and missing data. This campaign has many opportunities to learn and make changes to future campaigns in order to have a stronger positive impact. However, some limitations were found in our campaign:

1. Data collection was completed by different health staff and in few cases by volunteers, some of whom were not well experienced, and didn't attend the pre-campaign training; which resulted in some missing data and data inconsistency.

2. We have also noticed that the number of female was higher than male, $81 \%$ female and $19 \%$ male, this may be due to the fact that most males were at work during the time the sessions were conducted.

3. Also for the glycemic control, 2 hrs PPGT test can't be relied on because it's not very accurate most of the time, HbA1c is more accurate and efficient, but for budget constrains we didn't use this test.

\section{Conclusions and Recommendations}

Resources are limited within large health care systems such as UNRWA, so it is difficult to provide multiple weight management programs for specific subpopulations of patients with diverse disease conditions, especially when patients are scattered in a wide geographic radius. Utilizing existing NCD care resources to deliver the principles of effective behavior change in the areas of weight loss, healthy eating, physical activity, control the DM status, and prevention deterioration of the disease, would be more efficient in areas of time, cost and counseling.

In summary, we have found that this DM campaign focusing on healthy life style awareness by using a variety of sessions, improved body measurements and blood sugar in Palestine refugees with DM.

\section{However, we recommend the following:}

1. Such campaigns need to be sustained and expanded to other health centers.

2. Health education and spreading awareness about healthy lifestyle and how to control the blood sugar, should be systematical integrated in NCD UNRWA program.

3. Capacity building for the health staff, especially in the areas of NCD care management and improvement of their counseling skills should be rotationally done and monitored.

4. Local community involvement and NGOs partnerships have been strongly observed during the campaign activities, such relations should be sustain and empowered.

\section{Acknowledgement and Declarations}

None of the authors have a conflict of interest.Funded and supportedby the World Diabetes Foundation (WDF).

Acknowledgement goes to all UNRWA health staff who participated in the DM campaign across the four fields of operation (Jordan, Lebanon, West Bank and Gaza strip), also to the NGOs and Local communities in the four fields.

\section{References}

[1]. United Nations Relief and Works Agency for Palestine Refugees in the Near East (2015). http://www.unrwa.org/what-we-do

[2]. UNRWA (2013) Annual report of health department. http://www.unrwa. org/resources/reports/health-department-annual-report-2014

[3]. The International Diabetes Federation (IDF) (2014) Diabetes Atlas sixth Edition Poster update. https://www.idf.org/diabetesatlas/update-2014

[4]. Kyrou I, Kumar S (2010) Weight management in overweight and obese patients with type 2 diabetes mellitus. The British Journal of Diabetes \& Vascular disease 10(6): 274.

[5]. Gregg EW, Chen H, Wagenknecht LE, Clark JM, Delahanty LM, et al. (2012) Association of an Intensive Lifestyle Intervention with Remission of Type 2 Diabetes. Journal of American Medical Association 308(23): 24892496.

[6]. Shahin Y, Kapur A, Khader A, Zeidan W, Harries AD, et al. (2015) Clinical Audit on the Provision of Diabetes Care in the Primary Care Setting by United Nations Relief and Works Agency for Palestine Refugees in the Near East (UNRWA). Scientific Research 5(1): 12-20.

[7]. World Health Organization (WHO) (2008) Waist circumference and waisthip ratio: Report of a WHO expert consultation, Geneva. 8-11.

[8]. Brown AS (1990) Studies of Educational Interventions and Outcomes in Diabetic Adults: A Meta-Analysis Revisited. Patient Educ Couns 16(3): 189215.

[9]. Greenwood DA, Kramer MK, Hankins AI, Parise CA, Fox A, et al. (2014) Adapting the Group Lifestyle BalanceTM Program for Weight Management 
within a Large Health Care System Diabetes Education Program. Diabetes Educ 40(3): 299-307. http://tde.sagepub.com/content/40/3/299

[10]. Rickheim PL, Weaver TW, Flader JL, Kendall DM (2002) Assessment of Group Versus Individual Diabetes Education. A randomized study. Diabetes Care 25(2): 269-274.

[11]. Deakin T, McShane CE, Cade JE, Williams RD (2005) Group Based training for self-management strategies in people with Type 2 diabetes mellitus. Cochrane Database Syst Rev (2): CD003417

[12]. Sharon AB, Sandra U, Roberta A, Mary W, Gilbert R (1996) Promoting Weight Loss in Type II Diabetes. Diabetes Care 19(6): 613-624.
[13]. Vazquez G, Duval S, Jacobs DR Jr, Silventoinen K (2007) Comparison of Body Mass Index, Waist Circumference, an Waist/Hip Ratio in Predicting Incident Diabetes: A Meta-Analysis. Epidemiol Rev 29: 115-128.

[14]. Karl EF (2009) Waist Circumference Threshold Values for Type 2 Diabetes Risk. J Diabetes Sci Technol 3(4): 761-769.

[15]. Gómez-Pérez FJ (2015) Glycated Hemoglobin, Fasting, Two-hour Postchallenge and Postprandial Glycemia in the Diagnosis and Treatment of Diabetes Mellitus: Are We Giving Them the Right Interpretation and Use? Rev Invest Clin 67(2): 76-79. 